\title{
High order analysis of the limit cycle of the van der Pol oscillator
}

Paolo Amore, John P. Boyd, and Francisco M. Fernández

Citation: Journal of Mathematical Physics 59, 012702 (2018);

View online: https://doi.org/10.1063/1.5016961

View Table of Contents: http://aip.scitation.org/toc/jmp/59/1

Published by the American Institute of Physics

\section{Articles you may be interested in}

Dirac structures in nonequilibrium thermodynamics

Journal of Mathematical Physics 59, 012701 (2018); 10.1063/1.5017223

Newton's second law and the multiplication of distributions

Journal of Mathematical Physics 59, 013505 (2018); 10.1063/1.5021949

Generalized delta functions and their use in quantum optics

Journal of Mathematical Physics 59, 012102 (2018); 10.1063/1.4985938

Rigorous derivation of nonlinear Dirac equations for wave propagation in honeycomb structures Journal of Mathematical Physics 59, 011509 (2018); 10.1063/1.5021754

Generalized time-dependent Schrödinger equation in two dimensions under constraints Journal of Mathematical Physics 59, 012104 (2018); 10.1063/1.4996573

Noncommutative mapping from the symplectic formalism

Journal of Mathematical Physics 59, 012105 (2018); 10.1063/1.4986964

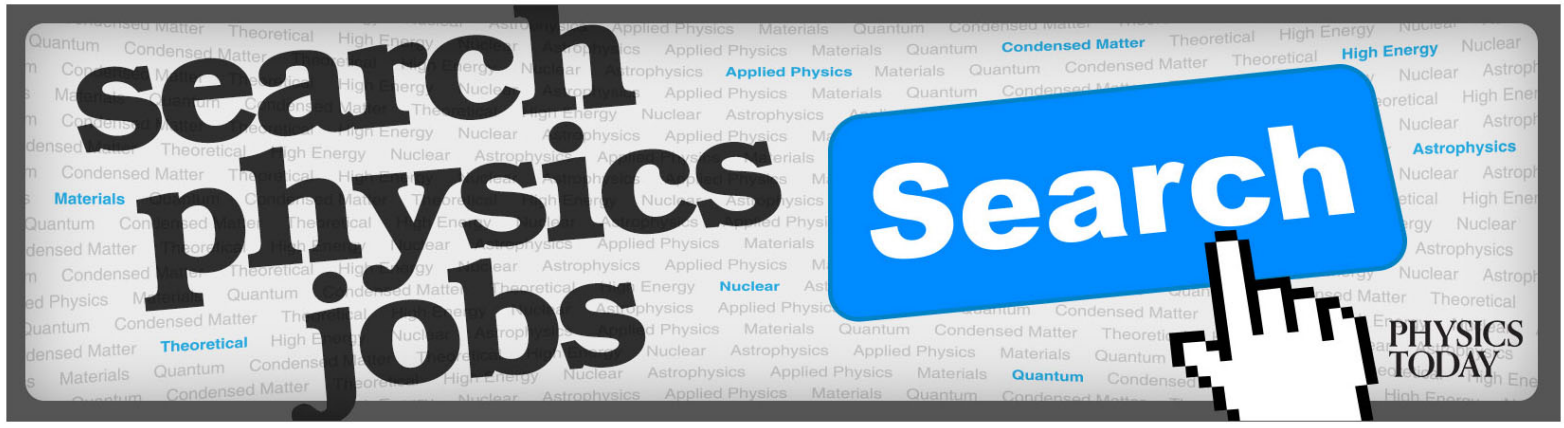




\title{
High order analysis of the limit cycle of the van der Pol oscillator
}

\author{
Paolo Amore, ${ }^{1, a)}$ John P. Boyd, ${ }^{2, b)}$ and Francisco M. Fernández ${ }^{3, c)}$ \\ ${ }^{1}$ Facultad de Ciencias, CUICBAS, Universidad de Colima, Bernal Díaz del Castillo 340, \\ Colima, Colima, Mexico \\ ${ }^{2}$ Department of Atmospheric, Oceanic and Space Science, University of Michigan, \\ 2455 Hayward Avenue, Ann Arbor, Michigan 48109, USA \\ ${ }^{3}$ INIFTA (CONICET), División Química Teórica, Blvd. 113 y 64 (S/N), Sucursal 4, \\ Casilla de Correo 16, 1900 La Plata, Argentina
}

(Received 22 November 2017; accepted 9 January 2018; published online 30 January 2018)

\begin{abstract}
We have applied the Lindstedt-Poincaré method to study the limit cycle of the van der Pol oscillator, obtaining the numerical coefficients of the series for the period and for the amplitude to order 859. Hermite-Padé approximants have been used to extract the location of the branch cut of the series with unprecedented accuracy (100 digits). Both series have then been resummed using an approach based on Padé approximants, where the exact asymptotic behaviors of the period and the amplitude are taken into account. Our results improve drastically all previous results obtained on this subject. Published by AIP Publishing. https://doi.org/10.1063/1.5016961
\end{abstract}

\section{INTRODUCTION}

The van der Pol equation is possibly the most known example of an ordinary differential equation with a limit cycle. It reads

$$
\ddot{x}(t)+x(t)=\mu \dot{x}(t)\left(1-x(t)^{2}\right),
$$

where $\mu>0$ is a parameter which controls the strength of the nonlinear contributions. Even though for $\mu=0$ this equation reduces to a simple harmonic oscillator, that can sustain oscillations of arbitrary amplitude, for any $\mu>0$, the van der Pol oscillator has a single oscillatory mode, with a specific amplitude and period, which depends on $\mu$. This mode is usually referred to as a "limit cycle." When the initial conditions are far from the limit cycle, the solution decays exponentially fast towards the limit cycle; this behavior results into a trajectory in phase space which spirals out (in) if the initial point falls inside (outside) the limit cycle, rapidly reaching the closed trajectory.

The reader may find a historical account of the origins of the van der Pol equation in a recent paper by Ginoux and Letellier; ${ }^{1}$ for a general perspective on the subject of self-sustained oscillations, it is useful to refer to the recent review article by Jenkins. ${ }^{2}$ Self-oscillations, as those described by Eq. (1), cover an important role in different areas of physics and mathematics, and even of biology: van der Pol and van der Mark, for example, described the heartbeat in terms of a relaxation oscillation and provided an electrical model of the heart; ${ }^{3}$ FitzHugh ${ }^{4}$ has generalized the van der Pol equation to describe the excitation of neurons and muscle fibers.

The task of finding approximate solutions to Eq. (1) becomes challenging, particularly when $\mu$ is large, because the system displays two alternating slow and fast regions, which reflect in a highly deformed cycle in phase space. We may classify the different strategies that have been used in the literature to solve the van der Pol equation into three main categories: numerical studies, carried out up to some large but finite value of $\mu$, asymptotic calculations, valid for $\mu \rightarrow \infty$, and perturbative calculations, obtained expanding about $\mu=0$.

\footnotetext{
a)Electronic mail: paolo.amore@gmail.com

b) Electronic mail: jpboyd@umich.edu

c) Electronic mail: framfer@gmail.com
} 
As nowadays, the numerical solution of Eq. (1) is easily accessible due to the wide disponibility of hardware and software, we mention the studies in the first category, ${ }^{5-8}$ mainly for historical reasons.

The first example of work belonging to the second class is given by Ref. 9, where a formula for the asymptotic period was given (as reported by Ginoux and Letellier ${ }^{1}$ )

$$
T^{(a s y m)}(\mu)=(3-\log 4) \mu+\frac{12.89}{\mu^{1 / 3}}+\frac{2}{\mu}\left(-3.31+\frac{19}{9} \log \mu\right)-\frac{4}{\mu^{5 / 3}}+\cdots .
$$

More precise formulas have then been derived in Refs. 6 and 10-12; the leading terms in the expression for the period read

$$
T^{(a s y m)}(\mu)=(3-\log 4) \mu-\frac{3 \alpha}{\mu^{1 / 3}}-\frac{2}{3 \mu} \log \mu-\frac{1.3246}{\mu^{2}}+\cdots,
$$

where $\alpha \approx-2.33810741$ is the highest zero of the Airy function.

An analogous formula for the amplitude of the oscillations has also been derived in Refs. 6, 10, and 11 and it reads

$$
A^{(\text {asym })}(\mu)=2-\frac{\alpha}{3 \mu^{4 / 3}}-\frac{16}{27} \frac{\log \mu}{\mu^{2}}+\frac{(3 \beta-1+\log 4-8 \log 3)}{9 \mu^{2}}+O\left(\mu^{-8 / 3}\right),
$$

where $\beta \approx 0.1723$.

Finally the last class has to do with the perturbative calculation of the solutions to Eq. (1): in this class falls the papers by Deprit and Rom ${ }^{13,14}$ and Deprit and Schmidt, ${ }^{15}$ by Andersen and Geer ${ }^{16}$ and Dadfar, Andersen and Geer, ${ }^{17}$ by Buonomo ${ }^{18}$ and, more recently, by Suetin. ${ }^{19}$ The Lindstedt-Poincaré method allows us to obtain expressions for the period and the amplitude as power series in $\mu$; the presence of a finite radius of convergence of these series, however, limits the direct application of the perturbative formulas to the domain of convergence. Different resummation procedures have been discussed in Refs. $16-19$, finding a radius of convergence $\mathcal{R} \approx 3.42$. In this class, we also mention Ref. 20, based on an alternative implementation of the Lindstedt-Poincare method, with improved convergence properties.

The Poincaré expansion theorem can be invoked to show that the periodic solution of the van der Pol equation can be expanded as a convergent power series for small values of the parameter $\epsilon{ }^{18,21}$

In the present work, we have set the following goals:

- To obtain the largest number of coefficients of the perturbative series for the amplitude and the period of the van der Pol oscillator;

- to obtain the most precise determination of the branch cut of these series;

- to perform a non-perturbative resummation of the power series, taking advantage of the goals previously achieved.

The paper is organized as follows: in Sec. II, we describe the Lindstedt-Poincaré method and compare our result with previously obtained results; in Sec. III, we apply the Hermite-Padé approximants to obtain a determination of an accurate location of the branch cut; the resummation of the series is carried out in Sec. IV; finally the conclusions are stated in Sec. V.

\section{LINDSTEDT-POINCARÉ METHOD}

We consider the van der Pol equation (1). As we have mentioned in the Introduction, this equation has a limit cycle, meaning that for $t \rightarrow \infty$ the solutions tend to a periodic solution with fixed amplitude, regardless of the initial conditions. In a straightforward application of perturbation theory, where the solution can be expressed as a power series in $\mu$, secular terms appear, which completely spoil the expansion.

In the Lindstedt-Poincaré method, one introduces a "universal" time $\tau=\Omega(\mu) t$ ("strained coordinate"), where $\Omega(\mu)$ is the exact frequency of the oscillations; the coefficients of the power series in $\mu$ for $\Omega$ are determined requiring that the "secular terms" arising at each perturbative order are 
avoided. In the particular case of the van der Pol oscillator, and more in general of problems with a limit cycle, a similar power series in $\mu$ must be assumed for the amplitude.

Let us briefly discuss in detail the implementation of the method for the van der Pol equation. After switching to the new time variable, we define $y(\tau) \equiv x(t)$ and the original differential equation takes the form

$$
\Omega^{2} \frac{d^{2} y}{d \tau^{2}}+y(\tau)-\mu \Omega \frac{d y}{d \tau}\left(1-y^{2}(\tau)\right)=0,
$$

with the initial conditions

$$
y(0)=\mathcal{A}(\mu) \quad ; \quad \frac{d y}{d \tau}(0)=0 .
$$

In our perturbative scheme, we will assume that

$$
\Omega(\mu)=1+\sum_{n=1}^{\infty} \omega_{n} \mu^{n}
$$

and

$$
\mathcal{A}(\mu)=\sum_{n=0}^{\infty} a_{n} \mu^{n},
$$

where $\omega_{n}$ and $a_{n}$ are constant coefficients and $\omega_{0}=1$ is the frequency of the linear system.

Similarly, we look for a solution

$$
y(\tau)=\sum_{n=1}^{\infty} y_{n}(\tau) \mu^{n}
$$

obeying the initial conditions

$$
y_{n}(0)=a_{n} \quad, \quad \frac{d y_{n}}{d \tau}(0)=0 .
$$

Upon substitution of these expressions in the differential equation, one has an infinite set of coupled linear non-homogeneous differential equations, corresponding to the different orders in $\mu$, which can be solved sequentially starting from the lowest order.

For instance to order $\mu^{0}$, one has

$$
\frac{d^{2} y_{0}}{d \tau^{2}}+y_{0}(\tau)=0
$$

with solution

$$
y_{0}(\tau)=a_{0} \cos \tau .
$$

To order $\mu$, using the solution of order 0 , one has the differential equation

$$
\frac{d^{2} y_{1}}{d \tau^{2}}+y_{1}(\tau)=\frac{a_{0}\left(a_{0}^{2}-4\right)}{4} \sin \tau+2 a_{0} \omega_{1} \cos \tau+\frac{a_{0}^{3}}{4} \sin 3 \tau,
$$

where in the last line the resonant terms containing $\sin \tau$ and $\cos \tau$ are responsible for the appearance of "secular terms" and therefore they must be avoided. This condition requires

$$
a_{0}=2 ; \omega_{1}=0 .
$$

The solution to the differential equation then takes the form

$$
y_{1}(\tau)=a_{1} \cos \tau+\frac{3}{4} \sin \tau-\frac{1}{4} \sin 3 \tau .
$$

Note that the first order solution contains the higher frequency 3.

The Lindstedt-Poincaré method can be efficiently implemented on a computer, to very large orders, to calculate the power series expansions for $\Omega, \mathcal{A}$, and $y$, either exactly or numerically. We have written two different programs, one for the symbolic calculation of the power series and the other for the numerical calculation, that have been performed working with 1000 digits.

In Table I, we compare the orders of the LP method obtained in previous studies with the orders obtained in the present work. The disposal of a large number of coefficients of the perturbative series, 
TABLE I. Comparison of the orders of the perturbative expansion for the van der Pol oscillator, obtained with the Lindstedt-Poincaré method on a computer, in different studies.

\begin{tabular}{lcc}
\hline \hline & Order (symbolic) & Order (numerical) \\
\hline Deprit and Rom $^{13}$ & $\ldots$ & $30(12$ digits $)$ \\
Deprit and Schmidt $^{15}$ & 8 & $\ldots$ \\
Andersen and Geer $^{16}$ & 24 & $163(37$ digits $)$ \\
Buonomo $^{18}$ & 120 & $402(40$ digits $)$ \\
Suetin $^{19}$ & $\ldots$ & $442(40$ digits $)$ \\
This work & 308 & $859(1000$ digits $)$ \\
\hline \hline
\end{tabular}

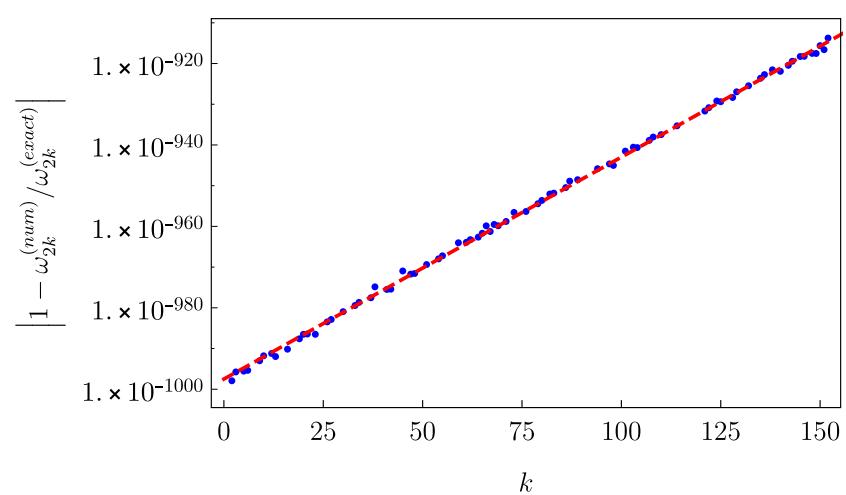

FIG. 1. Relative error over the numerical coefficients of the order $2 k$, with respect to the exact ones. The dashed line is the fit $10^{997.5-0.545 x}$

combined with the high accuracy of the calculation, will be essential for the numerical analysis performed in Secs. III-V.

To assess the quality of the numerical coefficients, in Fig. 1, we have plotted the quantity $-\log _{10}\left|1-\frac{\omega_{2 j}^{(\text {num })}}{\omega_{2 j}^{\text {(exact) }} \mid}\right|$, as a function of the order of the coefficients themselves. The dashed line in the plot is the linear fit

$$
f(x)=997.5-0.273 x,
$$

which vanishes at $x \approx 3660$ (thus $n_{\max } \approx 3660$ is approximately the highest order of the LindstedtPoincaré expansion that can be obtained working with 1000 digits).

\section{HERMITE-PADÉ APPROXIMANTS}

Applying the Lindstedt-Poincaré method as explained in Sec. II, one is able to obtain a large number of terms of the perturbative series for the period and for the amplitude of the van der Pol oscillator. Because these series are powers in the square of $\mu$, it is convenient to define the new expansion parameter $v \equiv \mu^{2}$. The period and amplitude series then take the form

$$
U_{N}=\sum_{n=0}^{N-1} c_{n} v^{n},
$$

where the coefficients $c_{n}$ are either calculated exactly or numerically, with high precision.

These series converge for $|v|<3.42[|\mu|<1.85]$ and diverge for larger $|v|$ where the radius of convergence is computed with high accuracy below.

Since arbitrarily large values of $v$ are of interest and calculations for $v$ as large as 10000 have been previously published, one is then faced with a task of extracting useful information from a series far beyond its radius of convergence. 
Suetin applied Padé approximation ${ }^{19}$ to analyze the singularities of the amplitude and frequency functions, building on work in Refs. 16 and 17. He conjectured ("it is possible that the following assertion is valid") the following:

1. The singularities of the amplitude and period functions are of the form

$$
g(v)\{\exp (i \alpha) \sqrt{v-\mathcal{R} \exp (i \phi)}+\exp (-i \alpha) \sqrt{v-\mathcal{R}}\}
$$

where $g(v)$ is holomorphic in a disk whose diameter is larger than $\mathcal{R}$.

2. The radius of convergence $\mathcal{R}$ is exactly 3.42 .

3. The phase $\phi$ is exactly $89 \pi / 156$.

4. $\alpha$ is $\pi / 4$ for the frequency and $3 \pi / 10$ for the amplitude.

However, Padé approximants are always rational functions and therefore are a clumsy tool for investigating square-roots. We therefore use a generalization of Padé approximations, HermitePadé approximants, which include square root singularities. Hermite-Padé approximations have been successfully used in rootfinding [Ref. 22, pp. 46-47 of Ref. 23], liquid drops ${ }^{24}$ and water waves, ${ }^{25}$ quantum mechanics, ${ }^{24,26-36}$ series analysis of multivalued functions, ${ }^{37}$ and other applications. ${ }^{22,24,26-37}$ The Cauchy rootfinding method employs a low order quadratic approximation. ${ }^{23} \mathrm{~A}$ Hermite-Padé approximation is defined as the solution to a polynomial equation of arbitrary userchosen degree. Here, we use only approximations that solve a quadratic equation. The name "Shafer approximation" is also used as a synonym for quadratic-solving Hermite-Padé approximations. ${ }^{22}$

Many illuminating and useful theorems have been proven about convergence properties of the Hermite-Padé approximants. However, numerical ill-conditioning is not unusual, so care must be taken and sometimes multiple precision arithmetic is helpful in achieving high accuracy.

To approximate a function $f(x)$ which is known only through the first $N$ term of its power series, use the function $f[K / L / M]$, the quadratic Shafer approximation, which is defined to be the solution of the quadratic equation,

$$
P(f[K / L / M])^{2}+Q f[K / L / M]+R=0,
$$

where the polynomials $P, Q$, and $R$ are of degrees $K, L$, and $M$, respectively. These polynomials are chosen so that the power series expansion of $f[K / L / M]$ agrees with that of $f$ through the first $N=K$ $+L+M+1$ terms.

The constant terms in $P$ and $Q$ can be set arbitrarily without loss of generality since these choices do not alter the root of the equation, so the total number of degrees of freedom is as indicated. As for ordinary Padé approximants, empirically, the most accurate approximations are usually obtained by choosing the polynomials to be of equal degree, the so-called "diagonal" approximants.

Again as for ordinary Padé approximants, the coefficients of the polynomials can be computed by solving a matrix equation. Cabay, Jones, and Labahn ${ }^{30}$ describe a faster but more ill-conditioned recursive algorithm to compute Hermite-Padé approximants. Mayer, Nuttall, and Tong present and apply a simple recursion restricted to the quadratic Shafer approximation. ${ }^{34,35}$ Loi and McInnes also offer an algorithm to compute the quadratic Hermite-Padé approximation. ${ }^{38}$

Like Padé approximants, Hermite-Padé approximants in a parameter $v$ are highly nonuniform in $v$. The approximations are extremely accurate for small $v$, but error grows exponentially with increasing $|v|$. Nevertheless, Hermite-Padé approximations are usually convergent far beyond the radius of convergence of the power series from which they were constructed. Indeed, they may converge exponentially fast for all finite $v$ even when the power series is factorially divergent for all non-zero $v$.

We applied classical Padé methods and stopped where these met our needs. When Padé methods disappointed, we shifted to Hermite-Padé.

Parenthetically, note that these power series based algorithms can be extended to Chebyshev interpolation as in the Hermite-Padé-Chebyshev work of Boyd. ${ }^{39}$ There is an extensive literature on 


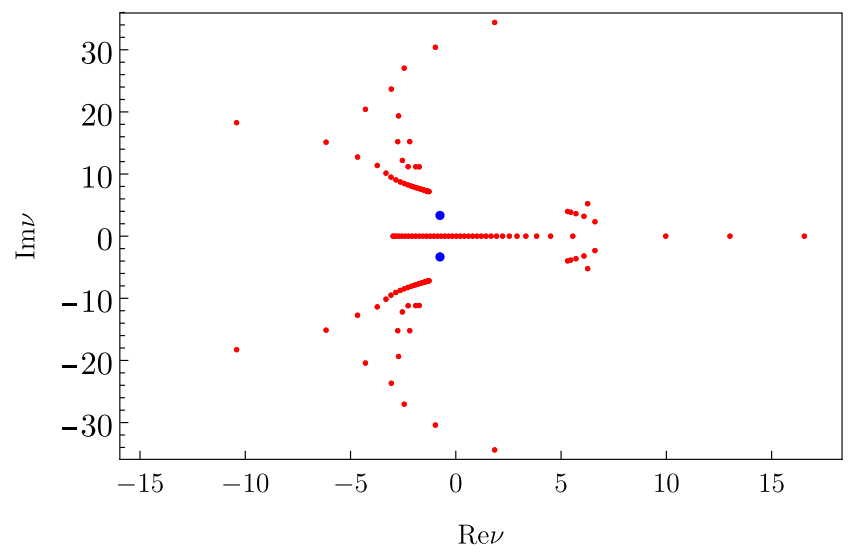

FIG. 2. Roots of $Q^{2}-4 P R$ for the case $K=L=M=142$; the blue ones correspond to the roots in the table.

Chebyshev polynomial generalizations of ordinary Padé approximations. ${ }^{40,41}$ The Chebyshev-based generalizations are much more uniform in accuracy on the interval spanned by the interpolation points than power series-based accelerations but cannot be applied directly to perturbation series. Rather, the Chebyshev-Padé algorithms are useful for converting numerical solutions at a set of discrete values of a parameter $v$ into spectrally accurate explicit approximations that are continuous in $v$.

In the complex $v$ plane, there are two complex conjugate roots,

$$
v_{ \pm}=\mathcal{R} e^{ \pm i \phi}
$$

at which $\Delta$ vanishes where $\Delta$ is the discriminant of (12), $\Delta=Q^{2}-4 P R$. The blue points in Fig. 2 correspond to these complex roots for the case $K=L=M=142$.

We have calculated the diagonal Hermite-Padé approximants up to a maximal order $K=142$, which corresponds to using 854 terms of the numerical series (where the coefficients of the series have been calculated with 1000 digits of accuracy). We have performed our analysis for both the series of the period and for the series of the amplitude: the plots in Fig. 3 illustrate the error over $\mathcal{R}$ and $\phi$ for both series, as a function of the order of the approximant. In Fig. 4, we plot the difference $\left|\mathcal{R}_{T}-\mathcal{R}_{A}\right|$, for the radius of convergence of the two series. The two results agree to at least 100 digits!

Our most accurate results are (where all digits are expected to be accurate)

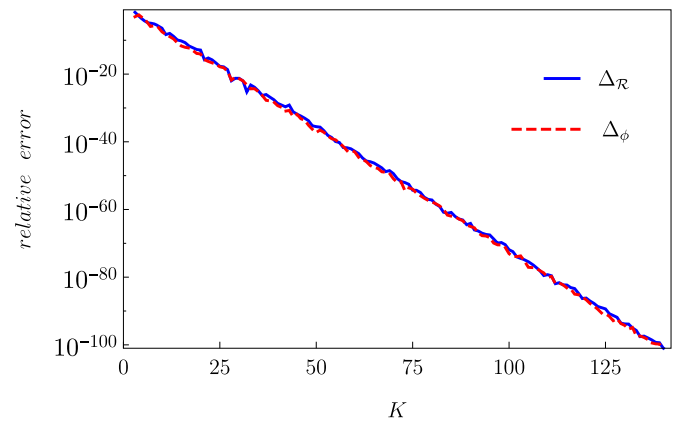

(a)

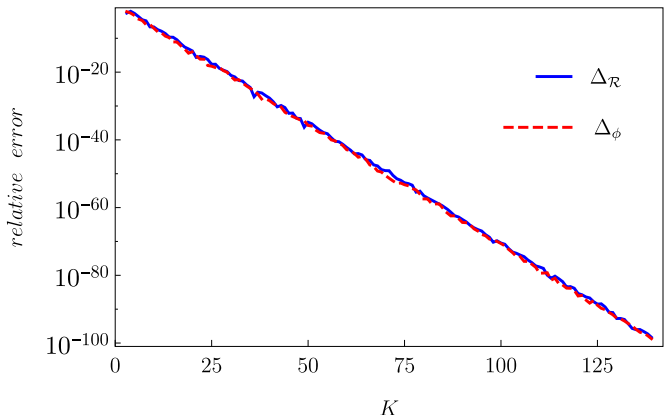

(b)

FIG. 3. Relative errors over the radius of convergence $\mathcal{R}$ and the phase $\phi$ estimated with the Hermite-Pade method for the series of the period (left plot) and for the series of the amplitude (right plot). Here $\Delta_{\mathcal{R}} \equiv\left|\mathcal{R}^{(K)}-\mathcal{R}^{(142)}\right|$ and $\Delta_{\phi} \equiv \mid \phi^{(K)}-$ $\phi^{(142)} \mid$. 


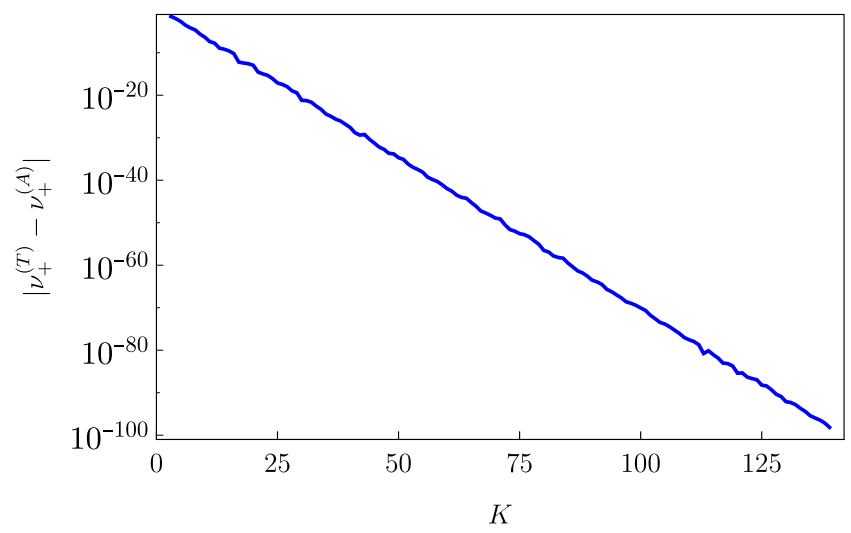

FIG. 4. $\left|v_{+}^{(T)}-v_{+}^{(A)}\right|$ as a function of the order $K$.

$$
\begin{aligned}
\mathcal{R}= & 3.420187909357135029477567375233640987583555906152748024797507314 \\
& 779205532661443624837516502144919691, \\
\phi= & 1.792288671545795214263603138015353292564791705087259065160879953 \\
& 197027910943997296402573377464202939 .
\end{aligned}
$$

These results falsify both Suetin's conjecture that $\mathcal{R}$ is exactly 3.42 and his suggestion that $\phi=89 \pi / 156$, which is in error by 0.0000302 .

Suetin mentions on p. S25 that he applied diagonal Hermite-Padé approximants to convince himself that the character of the singularities of the frequency and amplitude is more complicated than quadratic branching [square root singularities], but we disagree. A straightforward application of the generating function method developed by Fernández et al. ${ }^{42}$ that is a simplification of the approach proposed earlier by Hunter and Guerrieri ${ }^{43}$ confirms that the singularity is of square-root type.

The radical has many zeros besides the pair shown in blue in Fig. 2. The many spurious zeros can be identified by comparing calculations with different $K$. The relevant zeros of $R(v)$ are convergent whereas the spurious zeros hop around as the order $K$ of the Padé approximation is varied. This behavior is illustrated in Fig. 5, where we have plotted the minimal distance $d_{n}(K)$ of each of the roots of the Hermite-Padé approximant of order $K$ from any of the roots of order $K+1$, for $K=50$ (circles), $K=100$ (squares), and $K=138$ (triangles). It is evident that, while two of the roots converge to a given value (these are precisely the roots that we have mentioned earlier), the remaining roots do not converge.

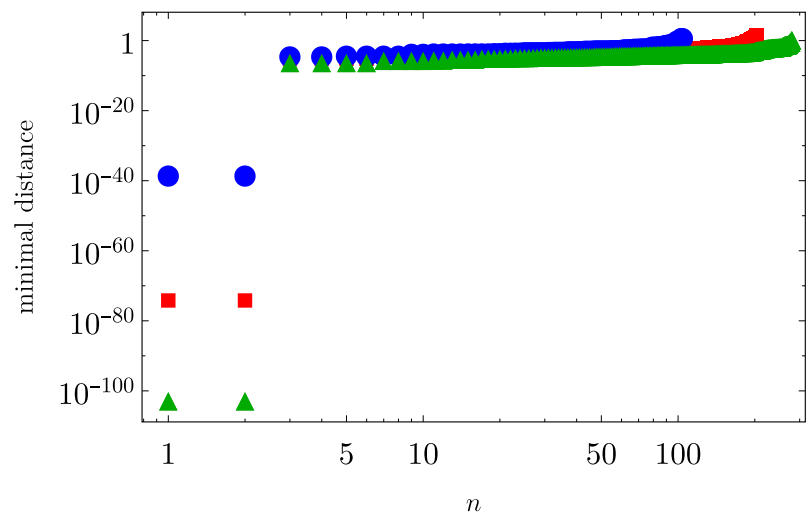

FIG. 5. Minimal distance $d_{n}(K)$ of each of the roots of the Hermite-Padé approximant of order $K$ ( $n$ is the index counting these roots) from any of the roots of order $K+1$, for $K=50$ (circles), $K=100$ (squares), and $K=138$ (triangles). 


\section{RESUMMATION}

The precise determination of the branch points of the series for the period and for the amplitude can be exploited to obtain an effective resummation of the series itself.

To this purpose, it is convenient to introduce the variable $\xi(v) \equiv\left(v^{2}-2 v \mathcal{R} \cos \phi+\mathcal{R}^{2}\right)^{1 / 4}$, which obeys the limit

$$
\lim _{v \rightarrow \infty} \frac{\xi(v)}{\sqrt{v}}=1
$$

On the other hand, the leading asymptotic behavior of the period, for $\mu \rightarrow \infty$, has been obtained by Dorodnitsyn ${ }^{10}$ [see Eq. (3)].

A simple strategy consists of "depurating" the period of the leading asymptotic behavior given above by introducing

$$
\begin{aligned}
\tilde{T}(v) & \equiv \frac{2 \pi}{\Omega}-(3-\log 4) \xi+\frac{3 \alpha}{\xi^{1 / 3}}+\frac{2 \log \xi}{3 \xi} \\
& +\left(1.3246+\frac{1}{2} \mathcal{R} \cos \phi(-3+\log 4)\right) \frac{1}{\xi}
\end{aligned}
$$

and then obtain the "complete" period as

$$
\begin{aligned}
T^{(\text {resum })} & =[\tilde{T}(v)]_{M, N}+(3-\log 4) \xi-\frac{3 \alpha}{\xi^{1 / 3}}-\frac{2 \log \xi}{3 \xi} \\
& -\left(1.3246+\frac{1}{2} \mathcal{R} \cos \phi(-3+\log 4)\right) \frac{1}{\xi},
\end{aligned}
$$

where $[\tilde{T}]_{M, N}$ is the $[M, N]$ Padé approximant to $\tilde{T}$. In the present case, since the asymptotic behavior up to order $1 / \mu$ has been taken care of, it is convenient to use $N=M+1$.

We can go a step further and try to guess the next contribution in the asymptotic formula; it makes sense to assume that this term behaves as $\gamma / \mu^{4 / 3}$, where $\gamma$ is a parameter, and therefore we write the "depurated" period as

$$
\begin{aligned}
\tilde{T}(v) & \equiv \frac{2 \pi}{\Omega}-(3-\log 4) \xi+\frac{3 \alpha}{\xi^{1 / 3}}+\frac{2 \log \xi}{3 \xi} \\
& +\left(1.3246+\frac{1}{2} \mathcal{R} \cos \phi(-3+\log 4)\right) \frac{1}{\xi}-\frac{\gamma}{\xi^{4 / 3}} .
\end{aligned}
$$

As before, we obtain the "complete" period as

$$
\begin{aligned}
T^{(\text {resum })}(\gamma) & =[\tilde{T}(v)]_{M, N}+(3-\log 4) \xi-\frac{3 \alpha}{\xi^{1 / 3}}-\frac{2 \log \xi}{3 \xi} \\
& -\left(1.3246+\frac{1}{2} \mathcal{R} \cos \phi(-3+\log 4)\right) \frac{1}{\xi}+\frac{\gamma}{\xi^{4 / 3}}
\end{aligned}
$$

In Fig. 6, we plot the relative error $\left|1-T^{(\text {resum })} / T^{(\text {num })}\right|$ at $\mu=100$, as a function of $\gamma$; the Padé approximant in the resummed expression is calculated using $M=N-1=120$. For $\gamma=0$, one recovers the results obtained earlier with the standard asymptotic formula (3). The error is minimal for $\gamma \approx-0.225$ (roughly two orders of magnitude better!).

The error $\left|1-T^{\text {(resum) }} / T^{(\text {num })}\right|$ is plotted in Fig. 7, using $T^{(\text {resum) }}(0)$ (solid line) and $T^{(\text {resum })}(-0.225)$ (dashed line). The resummed expression has been calculated using a Padé with $M=N-1=120$.

Let us now turn our attention to the amplitude. Equation (4) is the asymptotic expression for the amplitude for $\mu \rightarrow \infty$ derived by Dorodnitsyn and also confirmed later by Urabe $^{6}$ and by Ponzo and Wax. $^{11}$

As for the case of the period, we introduce a quantity where the leading asymptotic behaviors are eliminated

$$
\tilde{\mathcal{A}}(v) \equiv \mathcal{A}-2+\frac{\alpha}{3 \xi^{4 / 3}}-\frac{16}{27} \frac{\log \xi}{\xi^{2}}+\frac{1}{9 \xi^{2}}(3 \beta-1+\log 4-8 \log 3)
$$




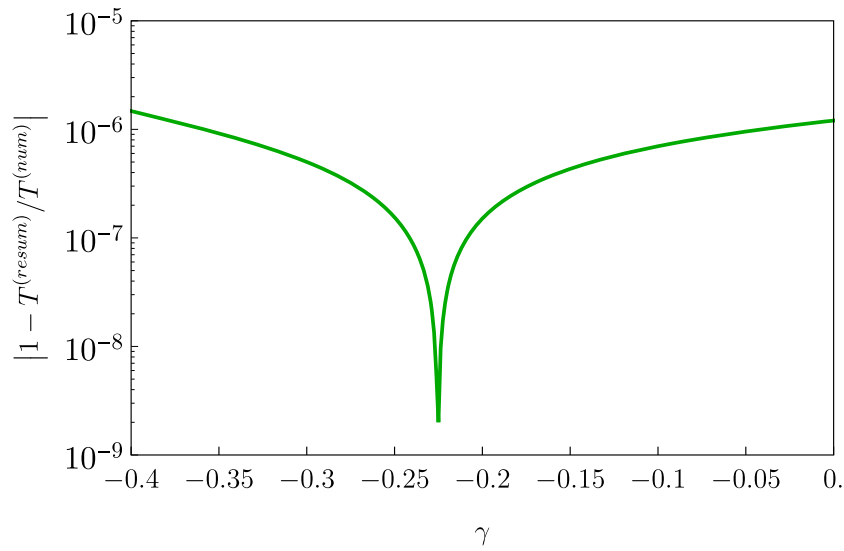

FIG. 6. Relative error $\left|1-T^{(\text {resum })} / T^{(\text {num })}\right|$ at $\mu=100$, as a function of $\gamma$.

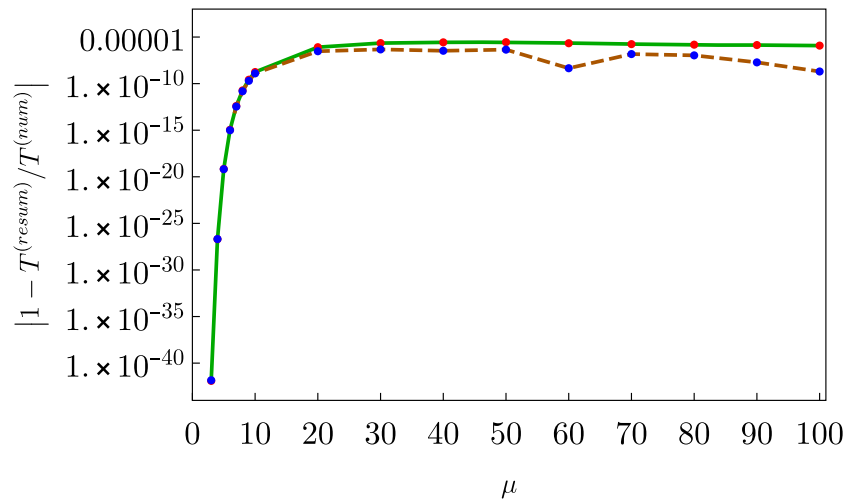

FIG. 7. Relative error over the period as a function of $\mu$ using $T^{(\text {resum })}(0)$ (solid line) and $T^{(\text {resum })}(-0.225)$ (dashed line). The resummed expression has been calculated using a Padé (not Hermite-Padé) with $M=N-1=100$.

and then obtain the "complete" amplitude as

$$
\begin{aligned}
\mathcal{A}^{(\text {resum })} & =[\tilde{\mathcal{A}}(v)]_{M, N}+2-\frac{\alpha}{3 \xi^{4 / 3}}+\frac{16}{27} \frac{\log \xi}{\xi^{2}} \\
& -\frac{1}{9 \xi^{2}}(3 \beta-1+\log 4-8 \log 3),
\end{aligned}
$$

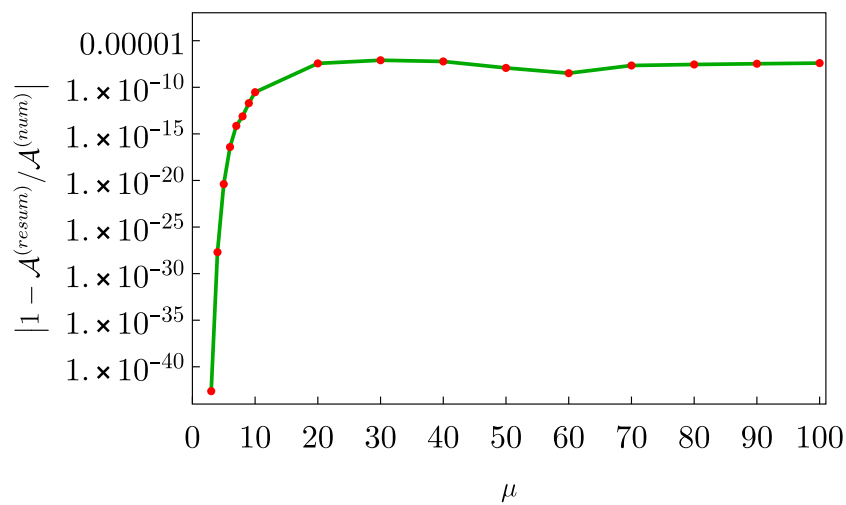

FIG. 8. Relative errors over the amplitude as a function of $\mu$ using $\mathcal{A}^{\text {(resum) }}$. The resummed expression has been calculated using a Padé (not Hermite-Padé) with $M=N-2=100$. 
where $[\tilde{\mathcal{A}}(v)]_{M, N}$ is the Padé approximant of orders $M$ and $N$. In the present case, it is convenient to choose $M=N-2$, given that the asymptotic formula (4) contains a term with the behavior $1 / \mu$. The relative error on the amplitude is shown in the plot of Fig. 8.

\section{CONCLUSIONS}

In this paper, we have applied the Lindstedt-Poincaré method to the van der Pol equation, to high orders in the expansion parameter. We have been able to reach order 308 in a purely symbolic calculation, where the perturbative coefficients are calculated in arbitrary precision, and order 859 in a numerical calculation, where the perturbative coefficients are calculated with an accuracy of 1000 digits. A comparison with similar calculations in the literature shows that our results are the most precise-most orders, most digits—(see Table I).

Using the precise coefficients both for the frequency (period) and for the amplitude, we have been able to estimate with unprecedented accuracy the radius of convergence $\mathcal{R}$ of the series and the location of the branch cut, using the Hermite-Padé approximant. In all the previous estimations, the radius of convergence and the phase ( $\mathcal{R}$ and $\phi$ respectively) had been obtained with few digits; for example, Andersen and Geer ${ }^{16}$ report $\mathcal{R} \approx 3.42$ and $\phi \approx 1.7925$, and a similar accuracy is also achieved by Suetin. ${ }^{19}$ Having at our disposal more (and more precisely calculated) series coefficients and using a different technique, the Hermite-Padé approximants, we have obtained results for $\mathcal{R}$ and $\phi$ which are expected to have the first 100 digits correct. It is remarkable that the numerical value of the branch cut for the series for the period and for the amplitude converge to the same result, within 100 digits.

This extremely accurate determination of the branch cut can be used to obtain a better resummation of the perturbative series. Our resummation involves a mapping of the perturbative parameter $\mu$ to $\xi(v)=\left(v^{2}-2 v \mathcal{R} \cos \phi+\mathcal{R}^{2}\right)^{1 / 4}$ (similar to the one used by Andersen and Geer), followed by a straightforward application of Padé approximants, taking into account the asymptotic behavior of the period and of the amplitude. The resummed expressions that we have obtained are much more accurate than similar expressions previously found (for a comparison see for instance ${ }^{16,18}$ ), over all range of $\mu$ considered.

\section{ACKNOWLEDGMENTS}

The research of P.A. was supported by the Sistema Nacional de Investigadores (México). J. P. Boyd was supported by the U.S. National Science Foundation through Grant No. DMS-1521158.

${ }^{1}$ J. M. Ginoux and C. Letellier, "Van der Pol and the history of relaxation oscillations: Toward the emergence of a concept," Chaos 22, 023120 (2012).

2 A. Jenkins, "Self-oscillation," Phys. Rep. 525, 167-222 (2013).

${ }^{3}$ B. van der Pol and J. van der Mark, "LXXII. The heartbeat considered as a relaxation oscillation, and an electrical model of the heart," Philos. Mag. 6(38), 763-775 (1928).

${ }^{4}$ R. FitzHugh, "Impulses and physiological states in theoretical models of nerve membrane," Biophys. J. 1, 445-466 (1961).

${ }^{5}$ W. S. Krogdahl, "Numerical solutions of the Van der Pol equation," Z. Angew. Math. Phys. 11(1), 59-63 (1960).

${ }^{6}$ M. Urabe, "Periodic solutions of van der Pols equation with damping coefficientCCCC0 10," IEEE Trans. Circuits Syst. 8, 382-386 (1960)

${ }^{7}$ C. W. Clenshaw, "The solution of van der Pol's equation in Chebyshev series," in Numerical Solution of Nonlinear Differential Equations, edited by D. Greenspan (Wiley, 1966), pp. 55-63.

${ }^{8}$ D. Greenspan, "Numerical approximation of periodic solutions of van der Pol's equation," J. Math. Anal. Appl. 39(3), 574-579 (1972).

${ }^{9}$ J. Haag, "Étude asymptotique des oscillations de relaxation (suite et fin)," Ann. Sci. Éc. Norm. Supér. 60, 65-111 (1943); "Étude asymptotique des oscillations de relaxation," Ann. Sci. Éc. Norm. Supér. 60, 289 (1943); "Exemples concrets d'étude asymptotique d'oscillations de relaxation," Ann. Sci. Éc. Norm. Supér. 61, 73-117 (1944).

${ }^{10}$ A. A. Dorodnitsyn, "Asymptotic laws of distribution of the characteristic values for certain special forms of differential equations of the second order," Uspekhi Math. Nauk 6(52), 3-96 (1952), 7.

${ }^{11}$ P. J. Ponzo and N. Wax, "On certain relaxation oscillations: Asymptotic solutions," J. Soc. Ind. Appl. Math. 13(3), 740-766 (1965).

12 J. A. Zonneveld, "Periodic solutions of the Van der Pol equation," Indagationes Math. 69, 620 (1966).

${ }^{13}$ A. Deprit and A. Rom, "Asymptotic representation of the cycle of Van der Pol's equation for small damping coefficients," Z. Angew. Math. Phys. 18, 736-747 (1967).

${ }^{14}$ A. Deprit and A. Rom, "Lindstedt's series on a computer," Astron. J. 73, 210-213 (1968). 
${ }^{15}$ A. Deprit and D. S. Schmidt, "Exact coefficients of the limit cycle in the van der Pol's equation,” J. Res. Natl. Bur. Stand. 84, 293-298 (1979).

${ }^{16}$ C. M. Andersen and J. F. Geer, "Power series expansions for the frequency and period of the limit cycle of the van der Pol equation,” SIAM J. Appl. Math. 42, 678-693 (1982).

${ }^{17}$ M. B. Dadfar, J. Geer, and C. M. Andersen, "Perturbation analysis of the limit cycle of the free van der Pol equation," SIAM J. Appl. Math. 44(5), 881-895 (1984).

${ }^{18}$ A. Buonomo, "The periodic solution of the van der Pol's equation," SIAM J. Appl. Math. 59, 156-171 (1998).

${ }^{19}$ S. P. Suetin, "Numerical analysis of some characteristics of the limit cycle of the free van der Pol equation," Proc. Steklov Inst. Math. 278(Suppl. 1), S1-S54 (2012).

${ }^{20} \mathrm{P}$. Amore and H. Montes, Phys. Lett. A 327, 158-166 (2004).

${ }^{21}$ F. Verhulst, Nonlinear Differential Equations and Dynamical Systems (Springer, Berlin, 2000).

${ }^{22}$ R. E. Shafer, “On quadratic approximation,” SIAM J. Numer. Anal. 11, 447-460 (1974).

${ }^{23}$ J. P. Boyd, Solving Transcendental Equations: The Chebyshev Polynomial Proxy and Other Numerical Rootfinders, Perturbation Series and Oracles (SIAM, Philadelphia, 2014), p. 460.

${ }^{24}$ M. Er-Riani, M. El Jarroudi, and O. Sero-Guillaume "Hermite-Padé approximation approach to shapes of rotating drops," Appl. Math. Modell. 38, 212-220 (2014).

${ }^{25}$ A. K. Common, "Applications of Hermite-Padé approximants to water waves and the harmonic oscillator on a lattice," J. Phys. A 15, 3665-3677 (1982).

${ }^{26}$ V. M. Vainnberg, V. D. Mur, V. S. Popov, and A. V. Sergeev, “Strong-field Stark effect,” JETP Lett. 44, 9-13 (1986).

${ }^{27}$ T. C. Germann and S. Kais, "Large order dimensional perturbation theory for complex energy eigenvalues," J. Chem. Phys. 99, 7739-7747 (1993).

${ }^{28}$ A. Natarov and J. P. Boyd, "Shafer (Hermite-Padé) approximants for functions with exponentially small imaginary part with application to equatorial waves with critical latitude," Appl. Math. Comput. 126, 109-117 (2002).

${ }^{29}$ M. López-Cabrera, D. Z. Goodson, D. R. Herschbach, and J. D. Morgan III, "Large-order dimensional perturbation theory for $\mathrm{H}_{2}^{+}$," Phys. Rev. Lett. 68, 1992-1995 (1992).

${ }^{30}$ S. Cabay, A. R. Jones, and G. Labahn, "Algorithm 766: Experiments with a weakly stable algorithm for computing Pade-Hermite and simultaneous Pade approximants," ACM Trans. Math. Software 23, 91-110 (1997).

${ }^{31}$ A. V. Sergeev, "Summation of the eigenvalue perturbation series by multivalued Padé approximants-Application to resonance problems and double wells," J. Phys. A: Math. Gen. 28, 4157-4162 (1995).

${ }^{32}$ A. A. Suvernev and D. Z. Goodson, "Perturbation theory for coupled anharmonic oscillators," J. Chem. Phys. 106, 2681-2684 (1997).

${ }^{33}$ A. V. Sergeev and D. Z. Goodson, "Summation of asymptotic expansions of multiple-valued functions using algebraic approximants: Application to anharmonic oscillators,” J. Phys. A: Math. Gen. 31, 4301-4317 (1998).

${ }^{34}$ I. L. Mayer and B. Y. Tong, "The quadratic Padé approximant method and its application for calculating densities of states," J. Phys. C: Solid State Phys. 18, 3297-3318 (1985).

${ }^{35}$ I. L. Mayer, J. L. Nuttall, and B. Y. Tong, "The quadratic Padé approximant method for calculating densities of states," Phys. Rev. B 29, 7102-7104 (1984).

${ }^{36}$ J. L. Gammel, "Generalized Padé approximations," in Pade Approximants and Their Applications, edited by P. R. GravesMorris (Academic Press, New York, 1973), pp. 3-9.

${ }^{37}$ P. J. Drazin and Y. Tourigny, "Numerical study of bifurcations by analytic continuation of a function defined by a power series,” SIAM J. Appl. Math. 56, 1-18 (1996).

${ }^{38}$ S. L. Loi and A. W. McInnes, "An algorithm for the quadratic approximation," J. Comput. Appl. Math. 11, 161 (1984).

${ }^{39}$ J. P. Boyd, "Chebyshev polynomial expansions for simultaneous approximation of two branches of a function with application to the one-dimensional Bratu equation," Appl. Math. Comput. 143, 189-200 (2002).

40 J. C. Mason and A. Crampton, "Laurent-Padé approximants to four kinds of Chebyshev polynomial expansions. Part I. Maehly type approximantions," Numer. Algorithms 38, 3-18 (2005).

${ }^{41}$ J. C. Mason and A. Crampton, "Laurent-Padé approximants to four kinds of Chebyshev polynomial expansions. Part II. Clenshaw-Lord type approximantions," Numer. Algorithms 38, 19-29 (2005).

${ }^{42}$ F. M. Fernández, G. A. Arteca, and E. A. Castro, "Singular points from Taylor series," J. Math. Phys. 28(2), 323-329 (1987)

${ }^{43}$ C. Hunter and B. Guerrieri, "Deducing the properties of singularities of functions from their Taylor series coefficients," SIAM J. Appl. Math. 39(2), 248-263 (1980). 